\title{
Effects of Medium Chain Triacylglycerols on the Pathological Condition and Energy Bioavailability of Streptozotocin-induced Diabetic Rats
}

\author{
Jun-ichi Nagata ${ }^{1 *}$, Chihiro Kuroiwa ${ }^{1}$, Shizuka Tamaru-Hase ${ }^{1,2}$ and Kazunori Koba ${ }^{2}$ \\ ${ }^{1}$ Department of Life, Environment and Materials Science, Fukuoka Institute of Technology, Fukuoka 811-0295, JAPAN \\ ${ }^{2}$ University of Nagasaki, Nagasaki 851-2195, JAPAN
}

\begin{abstract}
Diabetes is a disease characterized not only by a high blood glucose level but also by high ketone levels. Medium chain triacylglycerols (MCT) are known as functional lipids, but they are rapidly metabolized to produce ketone bodies. In this study, we examined the effects of MCT intake on the pathological condition and energy bioavailability of diabetic animals. Streptozotocin (STZ)-induced diabetic rats were used as animal models for type I diabetes. The pathological condition was estimated through blood biochemical values, and the energy expenditure was calculated based on the respiratory quotient, which was analyzed using a mass spectrometer.

Eight-week-old male STZ-induced diabetic Wistar rats were prepared and fed soybean oil (LCT) or MCT diets for 60 days. The survival rate of rats fed the MCT diet was higher than that of rats fed the LCT diet, although no ameliorating effects in the blood biochemical values were observed. In rats fed the LCT diet, their energy expenditure was much higher than their energy intake, whereas the energy balance was approximately equal in rats fed the MCT diet.

These results indicated that energy bioavailability may contribute, at least in part, to the high survival rates in the diabetic MCT group, although MCT intake did not definitely improve the pathological condition of the experimental rats.
\end{abstract}

Key words: medium-chain triacylglycerol, type 1 diabetic rat, survival rate, energy bioavailability

\section{INTRODUCTION}

Type 1 diabetes (T1D) is perceived as one of the most relevant autoimmune disorders in children. It is characterized by the destruction of the pancreatic $\beta$-cells that produce insulin $^{1-3)}$. Although this disorder is one of the well-characterized autoimmune diseases ${ }^{4}$, its mechanism of involvement in $\beta$-cell destruction is still unclear. In general, ß-cell autoantigens, macrophages, dendritic cells, B lymphocytes, and T lymphocytes are thought to be involved in the $ß$-cell specific autoimmune process ${ }^{5)}$. Extreme reduction in insulin secretion causes T1D. Since these patients cannot utilize carbohydrate, due to the dysfunction of the pancreatic ß-cells, they exclusively use fatty acids as their energy substrate ${ }^{6)}$. Consequently, these metabolic adaptations occur with ketosis or ketoacidosis in the patients with $\mathrm{T}^{\mathrm{D}} \mathrm{D}^{7}$. Besides, the global incidence and prevalence of $\mathrm{T} 1 \mathrm{D}$ have been increasing worldwide at a rate of nearly $3 \%$ per year ${ }^{8)}$. Moreover, T1D incidence is estimated to have increased more than 2 -fold in the past 20 years and is set to double again before $2020^{9)}$.

On the other hand, it is generally well known that medium-chain triglycerides (MCT), which consists of mediumchain fatty acids (MCFA), are remarkably different from long-chain triglycerides (LCTG) in terms of their absorption and metabolism. MCT are absorbed via the portal vein in the liver, more readily oxidized than LCTG, and used as an energy source without the carnitine transport system for mitochondrial entry ${ }^{10)}$. Moreover, MCT may have several advantages such as higher plasma clearance ${ }^{10)}$, lower trend to deposit as body fat ${ }^{11,12)}$, decrease in protein loss and im-

Abbreviations: MCT; Medium-chain triacylglycerol, LCT; Long-chain triacylglycerol, T1D; Type 1 diabetes, STZ; Streptozotocin, MCFA; Medium-chain fatty acid, AST; Aspartate transaminase, ALT; Alanine aminotransferase, A/G; Albumin globulin ratio, HSD; Honestly significant difference, RQ; Respiratory quotient, TG; Triacylglycerol

\footnotetext{
*Correspondence to: Jun-ichi Nagata, Department of Life, Environment and Materials Science, Fukuoka Institute of Technology, Fukuoka 811-0295, JAPAN

E-mail: j-nagata@ fit.ac.jp

Accepted November 27, 2017 (received for review September 21, 2017)
}

Journal of Oleo Science ISSN 1345-8957 print / ISSN 1347-3352 online

http://www.jstage.jst.go.jp/browse/jos/ http://mc.manusriptcentral.com/jjocs 
proved nitrogen-sparing effect ${ }^{13)}$ as compared with LCT. At present, cooking oil consisting of MCT is commercially available. However, high intake of MCT may induce a deficiency of essential polyunsaturated fatty acids and cause metabolic acidosis ${ }^{10,14)}$. Thus, MCFA have both physiological advantages and disadvantages.

Regarding diabetes, although there are some reports showing that MCT or MCFA is beneficial, there are some high-risk populations for ketogenesis, such as T1D patients. To date, there is little information concerning the pathological conditions caused by enteral feeding of MCT or MCFA in the population, such as in T1D patients.

The present study aimed to elucidate the effects of MCT in STZ-treated T1D rats. We attempted to clarify the impacts of MCT on the pathological condition of T1D animal model. We observed a dramatically high survival rate in T1D rats fed a MCT diet, as compared with that of T1D rats fed a LCT diet. Therefore, to explore the cause of the high survival rate of T1D rats fed a MCT diet, we examined the respiratory ratio for the assessment of the bioavailability and effectiveness of MCT as an energy substrate.

\section{EXPERIMENTAL}

\subsection{Animals and diets}

Male Wistar rats (180-200 g) were purchased from Japan SLC Inc. (Shidzuoka, Japan) and divided into 3 groups after a few days of adaptation based on body weight and blood glucose level: control $(n=5)$, diabetic $\operatorname{LCT}(n=7)$, and diabetic MCT $(n=6)$. The diabetes animal model was induced by a single intraperitoneal administration of a freshly prepared solution of STZ ( $50 \mathrm{mg} / \mathrm{kg}$ body weight) in $0.9 \%$ $\mathrm{NaCl}$. Control rats were injected with saline alone. The
Table 2 Fatty acid composition of dietary fats (wt\%).

\begin{tabular}{lcc}
\hline \multicolumn{1}{c}{ Fatty acids } & MCT & Soybean oil \\
\hline $8: 0$ & 71.9 & - \\
$10: 0$ & 24.0 & - \\
$12: 0$ & - & - \\
$14: 0$ & - & - \\
$16: 0$ & - & 10.7 \\
$16: 1$ & - & - \\
$18: 0$ & - & 1.96 \\
$18: 1$ & - & 28.4 \\
$18: 2$ & - & 54.9 \\
$18: 3$ (n-3) & - & 0.78 \\
Other (not identified) & 4.1 & 3.26 \\
\hline
\end{tabular}

MCT, medium-chain triacylglycerol.

animals were fed with an experimental diet, which was based on AIN-93G (Table 1) containing either soybean oil or a mixture of soybean oil and MCT for 60 days. The fatty acid composition of the dietary fats is shown in Table 2. These oils were kindly provided by The Nisshin OilliO Group, Ltd.

For monitoring of blood glucose levels, the blood levels after $6 \mathrm{~h}$ of fasting were determined using Accu-Chek Aviva blood glucose monitors (Roche Diagnostics, Indianapolis, IN, USA), by collecting blood via a tail cut in every 2 weeks until the 49th day of the experimental period. At the end of the experimental period, arterial blood was collected after $6 \mathrm{~h}$ of fasting to prepare plasma and stored at $-80^{\circ} \mathrm{C}$. The liver was immediately excised. The animal study was carried out under the Guidelines for Animal Experiments at the University of Nagasaki (Nagasaki, Japan) and under

Table 1 Composition of experimental diets.

\begin{tabular}{lrrrr}
\hline \multicolumn{1}{c}{$\begin{array}{c}\text { Ingredients } \\
\text { (g/kg diet) }\end{array}$} & Normal Wistar rats & & \multicolumn{2}{c}{ STZ-treated Wistar rats } \\
\cline { 2 - 2 } \cline { 5 - 6 } & LCT diets & & LCT diets & MCT diets \\
\hline Casein & 200 & & 200 & 200 \\
Soybean oil & 200 & & 200 & 100 \\
MLCT & 0 & & 0 & 100 \\
Sucrose & 250 & & 250 & 250 \\
Cellulose & 50 & & 50 & 50 \\
Mineral mixture (AIN93G) & 35 & & 35 & 35 \\
Vitamin mixture (AIN93) & 10 & & 10 & 10 \\
L-Cystine & 3 & & 3 & 3 \\
Choline bitartrate & 2.5 & & 2.5 & 2.5 \\
TBHQ & 0.014 & & 0.014 & 0.014 \\
$\alpha$-Cornstarch & 249.486 & & 249.486 & 249.486 \\
\hline
\end{tabular}


Law No. 105 and Notification No. 6 of the Government of Japan.

\subsection{Measurement of oxygen consumption and carbon di- oxide production.}

Open-circuit indirect calorimetry was performed with an $\mathrm{O}_{2} / \mathrm{CO}_{2}$ metabolism measuring system for small animals (ARCO-2000; Arco System, Chiba, Japan). The system monitored $\mathrm{VO}_{2}$ and $\mathrm{VCO}_{2}$ at 5-min intervals and calculated the respiratory quotient $(\mathrm{RQ})$ ratio $\left(\mathrm{VCO}_{2} / \mathrm{VO}_{2}\right)$. To measure the energy expenditure and spontaneous motor activity when sedentary, rats were individually placed in the chamber with an adequate amount of the experimental diet and tap water. The measurements of the energy expenditure under ad libitum conditions were performed for $24 \mathrm{~h}$ during the final week of the experimental period.

The substrate utilization rate and energy production rate were calculated using the formula used by Ferrannini ${ }^{15)}$, where the rate of glucose oxidation (g/min) was $4.55 \dot{\mathrm{V}} \mathrm{CO}_{2}$ (l/min) - $3.21 \dot{\mathrm{VO}}_{2}(\mathrm{l} / \mathrm{min})-2.87 \mathrm{~N}(\mathrm{mg} / \mathrm{min})$, the rate of lipid oxidation $(\mathrm{g} / \mathrm{min})$ was $\left(1.67 \dot{\mathrm{V}} \mathrm{O}_{2}-\dot{\mathrm{V}} \mathrm{CO}_{2}\right)-1.92 \mathrm{~N}$, and the rate of energy production (kcal/min) was $3.91 \dot{\mathrm{V}}_{2}+1.10$ $\dot{\mathrm{V}} \mathrm{CO}_{2}-3.34 \mathrm{~N}$. In this case, $\mathrm{N}$ was the rate of urinary nitrogen excretion used to estimate protein oxidation. However, considering that only a small portion of resting and exercise energy expenditure arises from protein oxidation ${ }^{15}$, the contributions of protein oxidation were neglected in this study.

In addition, the energy intake was expressed as the total energy intake, which was calculated based on the food intake and the substantial bioavailable energy intake, as estimated from the $\mathrm{RQ}$ values. The energy balance was showed as the difference between the substantial energy intake and the total energy expenditure.

\subsection{Analysis of blood biochemistry}

Plasma glucose and insulin concentrations of the rats were measured by the glucose CII test Wako kit (Wako Pure Chemicals Co. Ltd, Osaka, Japan) and Mercodia insulin ELISA kit for rat (Sapphire Bioscience, Pty, Ltd, Australia), respectively. Other blood biochemical parameters, such as the plasma cholesterol and triglyceride concentrations, urea nitrogen, creatinine, AST and ALT activities, and albumin-globulin ratio were analyzed enzymatically using the Cholesterol E-test Wako, Triglyceride E-test Wako, Urea nitrogen B-test Wako, Creatinine test Wako, Transaminase CII test Wako, and A/G B test Wako (Wako Pure Chemicals Co. Ltd, Osaka, Japan), respectively. In addition, the concentrations of the plasma ketone bodies were measured by a commercially available kit; Ketorex (Sanwa chemical Co., Tokyo, Japan).

\subsection{Statistical analysis}

Data were shown as an average or mean \pm SEM of the experimental rats. Statistical analysis was performed using the Log-Rank test for the survival rates of rats and the Tukey-Kramer HSD test was performed for other values, using JMP(Version 10.0.2 SAS Institute Inc., Cary, NC, USA). $P$ values $<0.05$ were considered significantly different between the groups.

\section{RESULTS}

3.1 Survival rate, growth parameters and relative tissue weights.

Survival rates of the normal rats and diabetic rats fed the MCT diet were 100\% through the end of the experimental period, while those of diabetic rats fed the LCT diet were approximately $30 \%$ or less. Survival rates of diabetic rats fed the LCT diet for 60 days were significantly lower $(p<$ $0.05)$ than the other groups. Unaccountably, all the diabetic rats fed the MCT diet survived throughout the entire experimental period (Fig. 1).

The time course changes of body weight during the experimental period are shown in Fig. 2. Body weights of diabetic rats fed the LCT diet were significantly lower $(p<$ 0.05) than those of normal rats fed the LCT diet from initial to the 57th day of the experimental period. Body weights of diabetic rats were maintained at a low level compared with those of normal rats throughout the experimental period.

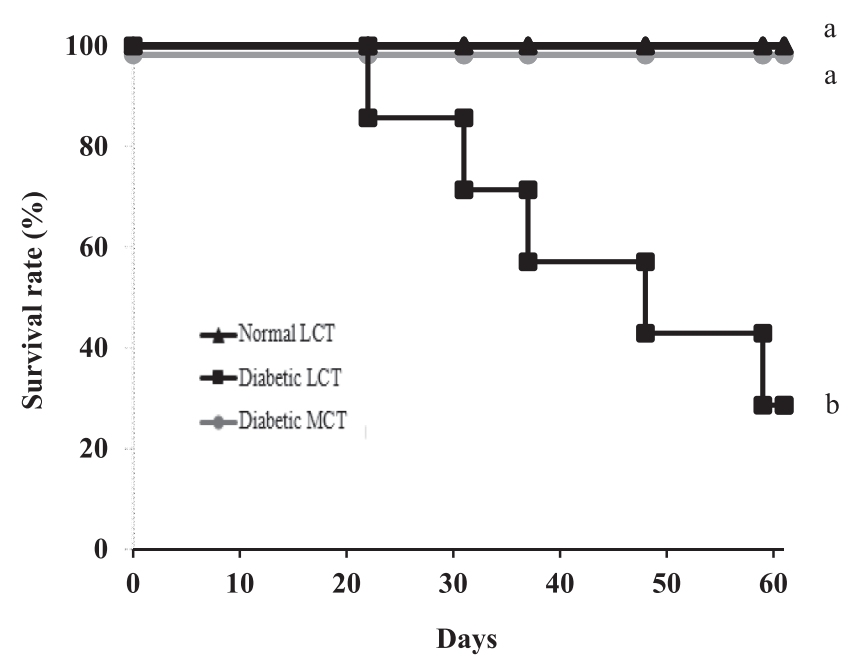

Fig. 1 Survival rate of normal and STZ-treated rats fed experimental diets for 60 days. Male Wistar rats were intraperitoneally administrated STZ at a dose of $50 \mathrm{mg} / \mathrm{kg}$ BW to establish T1D animal model. $\mathrm{T} 1 \mathrm{D}$ rats were fed the experimental diet containing either LCT or MCT. In addition, non-treated group as a control were fed the LCT diet. Different letters show the statistically significant differences $(p<$ 0.05) between each group. 


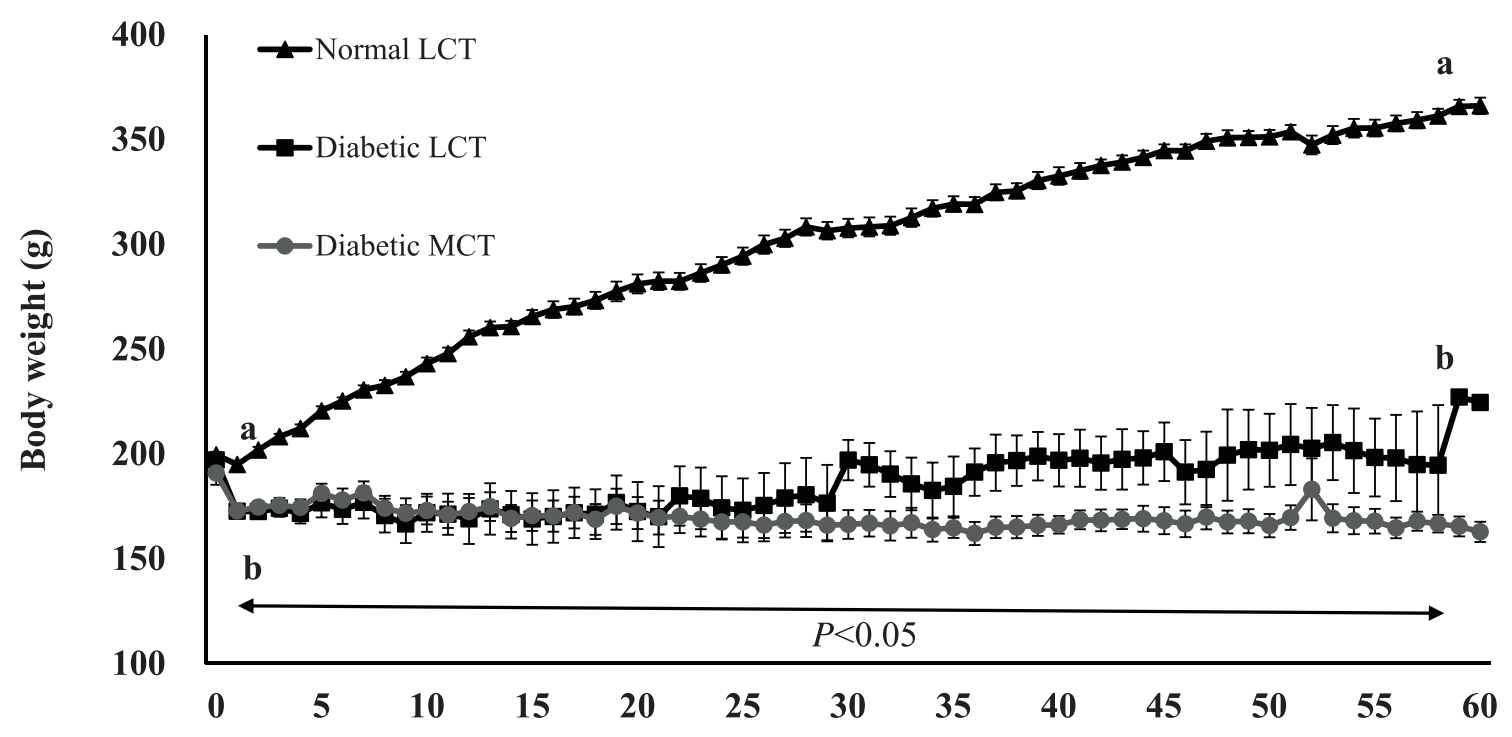

Days

Fig. 2 Time course changes of body weights of normal and STZ-treated rats fed experimental diets throughout the experimental period. Rats were weighed every day during the experimental period. Each point represents the mean $\pm \mathrm{SEM}$ in the case of 3 or more survivals from the start of experiment up to 57 days. After that day, statistical comparison was not conducted because there were 2 T1D rats in LCT group. Different letters show the significant differences $(p<0.05)$ of control ver. T1D rats fed the LCT diets.

Relative liver and kidney weights of diabetic rats were approximately 1.5 and 2 to 3 times greater than those of normal rats, respectively. Weights of adipose tissues, such as perirenal, epididymal, and mesenteric adipose tissues in diabetic model rats fed the MCT diet were the smallest among the groups (Table 3).

\subsection{Blood biochemical values}

Time course changes of blood glucose levels of rats during the experimental period are illustrated in Fig. 3. Blood glucose concentrations of T1D rats fed the LCT diet were significantly higher $(p<0.05)$ than those of normal rats fed the LCT diet during the experimental period. In addition, time course changes of blood glucose levels of diabetic rats fed the MCT diet were kept higher than those of normal rats fed the LCT diet(Fig. 3).

Final blood biochemical values are listed in Table 4. Triacylglycerol concentrations of diabetic rats fed the MCT diet were extremely higher than those of the other animals. Their values were approximately 8-fold higher than those of normal rats. This indicates that feeding diabetic rats with MCT caused hypertriglycemia. However, the TG concentrations of diabetic rats fed the LCT diet were not as high as those of rats fed the MCT diet. Other biochemical values, such as the liver and kidney function indices, were higher in the diabetes model rats fed the MCT diet relative to those of normal rats. In addition to these indices, blood glucose concentrations of the diabetes model rats fed the MCT diet were also the highest among the experimental groups.

On the other hand, ketone body concentrations of diabetic model rats were not markedly affected by MCT feeding. Insulin levels of diabetic model rats were depressed by STZ treatment.

These results almost agreed with the results of our previous study, which was carried out with five animals per group. In particular, reproducibility of survival rate, blood TG and ketone body concentrations, and liver function index value were confirmed (data not shown).

\subsection{Respiratory analysis for measurement of energy ex- penditure and respiratory quotient}

Daily total energy expenditure, respiratory quotient (RQ) and energy balance are listed in Table 5. The daily total energy expenditure of diabetic rats fed the LCT diet was comparable with that of normal rats, while the daily total energy expenditure of diabetic rats fed the MCT diet was significantly lower than that of diabetic rats fed the LCT diet. From the respiratory analysis, the RQ values of normal rats fed the LCT diet and diabetic rats fed either the LCT or the MCT diets were approximately $0.77,0.685$ and 0.685 , respectively. Although the T1D rats consumed energy exclusively from dietary fats, the normal rats consumed it from dietary fats and carbohydrate in an energy 
Table 3 Body weights, food intake and organ weights of rats fed experimental diets.

\begin{tabular}{lcccr}
\hline & Normal Wistar rats & & \multicolumn{2}{c}{ STZ-treated Wistar rats } \\
\cline { 2 - 2 } & LCT diets & & LCT diets & MCT diets \\
\hline $\begin{array}{l}\text { Initial body weight }(\mathrm{g}) \\
\text { Final body weight }(\mathrm{g})\end{array}$ & $200 \pm 1.5$ & & $194.2 \pm 2.0$ & $197 \pm 1.8$ \\
& $361 \pm 4.0$ & & 226.8 & $161.6 \pm 5.4$ \\
$\begin{array}{l}\text { Food intake (g/day) } \\
\begin{array}{l}\text { Food efficiency } \\
\text { (g BW/g diet) }\end{array}\end{array}$ & $0.17 \pm 0.01$ & & 22.4 & $19.9 \pm 0.9$
\end{tabular}

Tissue weight

(g/100 g BW)

Liver weight

$2.97 \pm 0.08$

4.32

$4.60 \pm 0.09$

Kidney weight

$0.58 \pm 0.00$

1.30

$1.80 \pm 0.06$

Adipose tissues

(g/100 g BW)

$\begin{array}{llll}\text { Epidydimal } & 3.72 \pm 0.15 & 1.83 & 0.81 \pm 0.13 \\ \text { Perirenal } & 2.94 \pm 0.11 & 1.22 & 0.20 \pm 0.07 \\ \text { Mesenteric } & 2.85 \pm 0.13 & 0.87 & 0.22 \pm 0.05\end{array}$

Data were shown as an average of 2 rats or Mean \pm SEM of $5-6$ rats.

After the 57 th day of experiment, statistical comparison was not conducted because there were 2 T1D rats in LCT group.

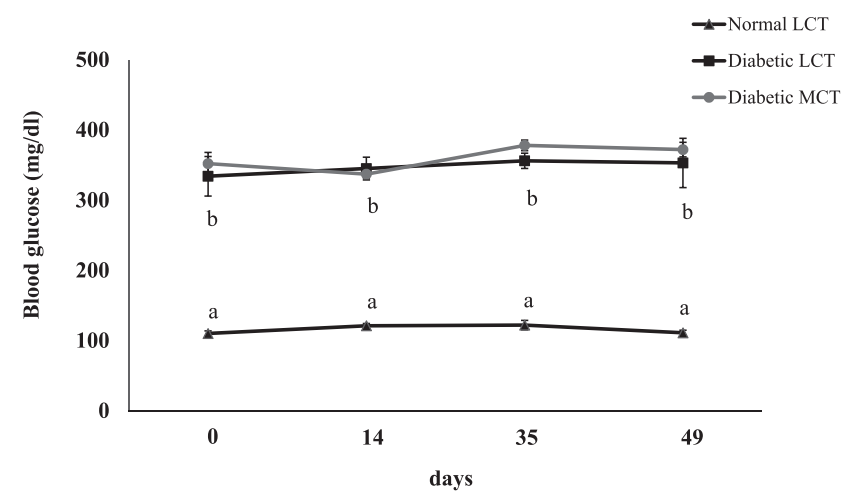

Fig. 3 Time course changes of blood glucose levels of normal and STZ-treated rats fed experimental diets. Blood glucose levels of STZ-treated rats were significantly higher $(p<0.05)$ than that of normal rats throughout the experimental period. The data are shown as the mean \pm SEM in the case of 3 or more survivals from the start of experiment up to 49 days. Different letters show the significant differences $(p<0.05)$ of control ver. T1D rats fed the LCT diets.

ratio of 3 to 1 . The energy balance of T1D rats fed the MCT diet was almost equal $(-0.2(\mathrm{kcal} /$ day $))$, but that of T1D rats fed the LCT diet exhibited a negative balance $(-4.2$ $(\mathrm{kcal} /$ day $))$.

\section{DISCUSSION}

In the present study, we examined the effects of MCT on the pathological condition and energy bioavailability in STZ-induced T1D rats. As a result, we found that the MCT intake may contribute to the high survival rates and produce vital life energy. From the blood biochemical values, however, MCT intake did not necessarily indicate an improvement of the pathological condition in T1D rats. Moreover, the results from the respiratory gas analysis showed that MCT could slightly be utilized as an energy source compared with LCT.

$\mathrm{T} 1 \mathrm{D}$ is the kind of disorder that presents not only with hyperglycemia but also with ketosis as a characteristic of the disease ${ }^{16)}$. Since this type of diabetic prevents the use of carbohydrates as an energy substrate, fatty acids composed of triacylglycerol are exclusively the energy source, resulting in the production of a large amount of ketone bodies. Severe ketosis seems to be responsible for metabolic acidosis ${ }^{17)}$. Overwhelming ketoacidosis potentially causes fatal syndromes such as a diabetic coma ${ }^{18)}$.

MCT is well known to rapidly metabolize and easily produce ketone bodies compared with LCT. Since the 
Table 4 Blood biochemical values of the experimental rats.

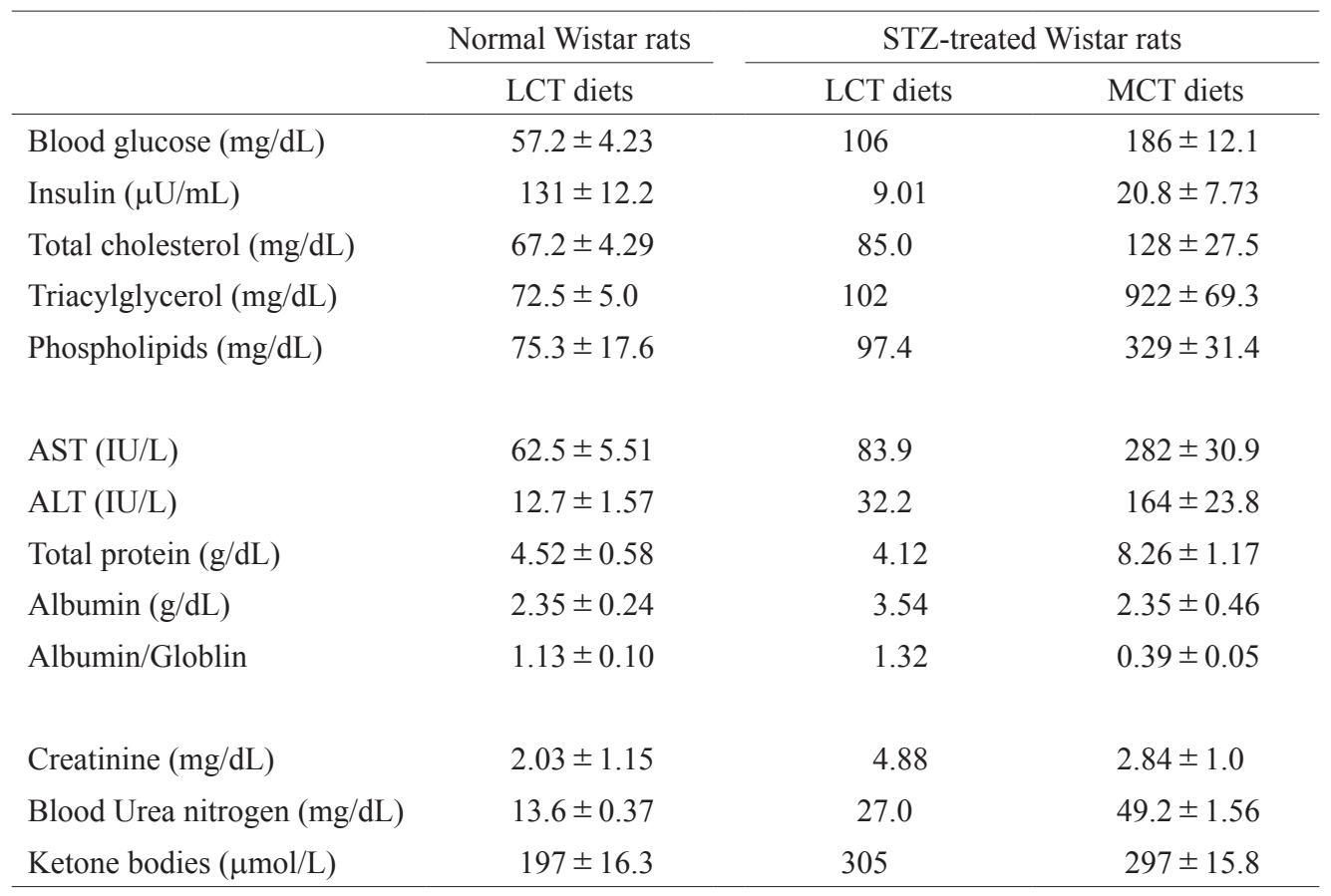

Data were shown as an average of 2 rats or Mean \pm SEM of $5-6$ rats.

After the 57th day of experiment, statistical comparison was not conducted because there were 2 T1D rats in LCT group.

Table 5 Energy balance and Respiratory quotient $(\mathrm{RQ})$ of normal and T1D rats fed experimental diets.

\begin{tabular}{lccccc}
\hline & Normal Wistar rats & & \multicolumn{2}{c}{ STZ-treated Wistar rats } \\
\cline { 2 - 2 } \cline { 5 - 5 } \cline { 5 - 6 } & LCT diets & & LCT diets & MCT diets \\
\hline Food intake (g/day) & $16.4 \pm 0.98^{\mathrm{A}}$ & & $23.2 \pm 4.7^{\mathrm{B}}$ & $20.6 \pm 1.07$ \\
Total energy intake (kcal/day) & $75.2 \pm 4.50^{\mathrm{A}}$ & & $107 \pm 4.6^{\mathrm{B}}$ & $94.7 \pm 4.92$ \\
Bioavailable energy intake (kcal/day) & $62.1 \pm 3.72^{\mathrm{A}}$ & & $41.7 \pm 1.79^{\mathrm{B}}$ & $37.1 \pm 1.93$ \\
$\quad$ Fat (kcal/day) & $29.4 \pm 1.76^{\mathrm{A}}$ & & $41.7 \pm 1.79^{\mathrm{B}}$ & $37.1 \pm 1.93$ \\
$\quad$ Carbohydrate (kcal/day) & $32.7 \pm 1.96^{\mathrm{A}}$ & & $0.0 \pm 0.00^{\mathrm{B}}$ & $0.0 \pm 0.00$ \\
RQ & $0.77 \pm 0.01$ & & $0.69 \pm 0.00$ & $0.69 \pm 0.00$ \\
& & & & \\
Total energy expenditure (kcal/day) & $45.7 \pm 1.02$ & & $45.9 \pm 4.46^{\mathrm{a}}$ & $37.3 \pm 1.75^{\mathrm{b}}$ \\
$\quad$ Fat (kcal/day) & $33.3 \pm 1.65^{\mathrm{A}}$ & & $45.9 \pm 4.46^{\mathrm{B}}$ & $37.3 \pm 1.75$ \\
$\quad$ Carbohydrate (kcal/day) & $12.3 \pm 2.5^{\mathrm{A}}$ & & $0.0 \pm 0.00^{\mathrm{B}}$ & $0.0 \pm 0.00$
\end{tabular}

Energy balance (kcal/day)

( = bioavailable intake-expenditure)

$16.4 \pm 4.12^{\mathrm{A}}$

$-4.20 \pm 2.72^{\mathrm{B}} \quad-0.20 \pm 1.01$

Data were shown as Mean \pm SEM of 3 to 6 rats. Tukey-Kramer HSD' test, A, B $p<0.05$ : Normal rats fed LCT diets vs. STZ-rats fed LCT diets. a, b $p<0.05$ : STZ-rats fed LCT diets vs. STZ-rats fed MCT diets.

Except for bioavailable energy intake which is the average of 57-day intake, the measurements of energy expenditure under ad libitum conditions were performed for $24 \mathrm{~h}$ at the final week of experimental period. 


\section{Effects of MCT in Type 1 Diabetic Rats}

feeding of MCT in diabetes is concerned with the possibility of a marked elevation in the blood ketone body concentration $^{19)}$, MCT is generally thought of as a food stuff that diabetic patients should pay attention. However, in this study, we observed a high survival rate of rats fed MCT diet throughout the experimental period. On the other hand, the survival rate of rats fed the LCT diet was less than 30\% $(2 / 7)$ at the end of the experiment. There was a significant difference in the survival rate between the LCT group and the other groups (Fig. 1). In addition, we assume that the number of deaths in T1D rats fed the LCT diet was high, but not dependent on the ketone body concentration. To elucidate how MCT intake contributes to a high survival rate, we focused on the improvement of pathological conditions and the energy bioavailability in T1D rats.

Blood biochemical values, such as the blood glucose, liver and renal function values of rats fed the MCT diet did not show any significant improvements. These results mean that MCT intake did not improve T1D. Moreover, feeding of MCT even characteristically increased serum TG and ketone body levels in the T1D animal model. In general, it is known that patients with diabetes exhibit triglyceridemia and deterioration of liver and renal functions based on the severity of the diabetes ${ }^{20)}$. Therefore, in the present study, we assumed that the MCFA were hydrolyzed and absorbed but not fully utilized in T1D rats, resulting in with and without elevated serum TG and ketone body concentrations. These results indicate that the alteration of the pathological condition in the T1D animal models may not be associated with the survival rate of T1D rats fed the MCT diet.

Based on the result of the RQ ratio, it was readily considered that the both LCT and MCT were utilized as the main energy source in the current animal models. Therefore, we examined the energy balance on the basis of the difference in energy consumption and intake, because we assumed that the significant differences in the survival rate of the diabetic model rats fed the LCT and the MCT diets might be attributed to the bioavailability of dietary fat. As shown in Table 5, although both LCT and MCT intake in T1D rats showed a negative energy balance, the value of the negative energy balance of diabetic rats fed the LCT diet was approximately 5-fold that of the MCT diet group and accounted for about $15 \%$ of the energy consumption in rats fed the LCT diet. However, the negative energy balance of diabetic rats fed the MCT diet was approximately $4 \%$ of the energy consumption. MCT is expected to be exploited as the energy substrate for the minimum required amount for survival, even in the severe diabetic condition, since nutrients such as protein and carbohydrates cannot be used effectively. In the present study, the results of the blood ketone body and TG concentrations of T1D rats fed the MCT diet do not clearly indicate that MCT was effectively utilized as the energy substrate in the T1D animal model. Therefore, it is likely that there is excess MCT or MCFA circulating in their blood. Thus, the slight difference in the utilization of dietary fat as an energy substrate may lead to higher survival rates in rats fed the MCT diet.

\section{CONCLUSION}

We examined the pathological condition, such as the ketone bodies, blood glucose and insulin concentrations, and energy balance in a T1D animal model fed a MCT diet for 60 days. We found a significantly higher survival rate in $\mathrm{T} 1 \mathrm{D}$ rats fed the MCT diet by the slight contribution toward the energy bioavailability, without the increase in blood ketone body levels, compared with those of the animal model fed a LCT diet. However, we could not detect any alterations in the pathological conditions. These results suggest that MCT intake in the T1D animal model supplies, at least in part, the minimum amount of the energy substrate required to maintain their life, although pathological improvements of diabetes cannot be expected. Further studies are required to elucidate the possible factors that affect the higher survival rate induced by MCT intake.

\section{Acknowledgements}

This research was partially supported by the Ministry of Education, Science, Sports and Culture of Japan, Grant-inAid for Scientific Research (C), 2012-2016(24501027, Junichi Nagata).

\section{References}

1) Todd, J.A. Etiology of type 1 diabetes. Immunity 32 , 457-467 (2010).

2) Bluestone, J.A.; Herold, K.; Eisenbarth G. Genetics, pathogenesis and clinical interventions in type 1 diabetes. Nature 464, 1293-1300 (2010).

3) Atkinson, M.A.; Eisenbarth, G.S.; Michels, A.W. Type 1 diabetes. Lancet 383, 69-82 (2014).

4) van Belle, T.L.; Coppieters, K.T.; Von Herrath, M.G. Type 1 diabetes: Immunology, and therapeutic strategies. Physiol. Rev. 91, 79-118(2011).

5) Yoon, J.W.; Jun, H.S. Autoimmune destruction of pancreatic beta cells. Am. J. Ther. 12, 573-579(2005).

6) Wohl, P.; Girman, P.; Pelikánová, T. Inflexibility of energy substrate oxidation in type 1 diabetic patients. Metabolism 53, 655-659(2004).

7) Lebovitz, H.E. Diabetic ketoacidosis. Lancet 25, 767772 (1995)

8) Onkamo, P.; Väänänen. S.; Karvonen, M.; Tuomilehto, J. Worldwide increase in incidence of Type I diabetes-the 
analysis of the data on published incidence trends. $D i$ abetologia 42, 1395-1403(1999).

9) Odegaard, J.I.; Chawla, A. Connecting type 1 and type 2 diabetes through innate immunity. Cold Spring Harb Perspect Med. 2, a007724 (2012).

10) Papamandjaris, A.A.; MacDougall, D.E.; Jones, P.J. Medium chain fatty acid metabolism and energy expenditure: obesity treatment implications. Life Sci. 62, 1203-1215 (1998).

11) Tsuji, H.; Kasai, M.; Takeuchi, H.; Nakamura, M.; Okazaki, M.; Kondo, K. Dietary medium-chain triacylglycerols suppress accumulation of body fat in a doubleblind, controlled trial in healthy men and women. $J$. Nutr. 131, 2853-2859 (2001).

12) Takeuchi, H.; Sekine, S.; Kojima, K.; Aoyama, T. The application of medium-chain fatty acids: ed1ible oil with a suppressing effect on body fat accumulation. Asia Pac. J. Clin. Nutr. Suppl. 1, 320-323(2008).

13) Bach, A.C.; Frey, A.; Lutz, O. Clinical and experimental effects of medium-chain-triglyceride-based fat emulsions-a review. Clin. Nutr. 8, 223-235(1989).

14) Pettei, M.J.; Daftary, S.; Levine, J.J. Essential fatty acid deficiency associated with the use of a mediumchain-triglyceride infant formula in pediatric hepatobiliary disease. Am. J. Clin. Nutr. 53, 1217-1221(1991).

15) Ferrannini, E. The theoretical bases of indirect calorimetry: A review. Metabolism 37, 287-301 (1988).

16) American Diabetes Association. Diagnosis and classification of diabetes mellitus. Diabetes Care 32 (Suppl.1), S62-67 (2009).

17) Stojanovic, V.; Ihle, S. Role of beta-hydroxybutyric acid in diabetic ketoacidosis: A review. Can. Ver. J. 52, 426-430 (2011).

18) Kety, S.S.; Polis, B.D.; Nadler, C.S.; Schmidt, C.F. The blood flow and oxygen consumption of the human brain in diabetic acidosis and coma. J. Clin. Invest. 27, 500-510 (1948).

19) Bach, A.C.; Babayan, V.K. Medium-chain triglycerides: An update. Am. J. Clin. Nutr. 36, 950-962 (1982).

20) Yoshitsugu, M.; Iwase, T.; Sassa, R. Effects of obesity, hyperlipidemia and impaired glucose tolerance on biochemical liver function tests. Nippon Shokakibyo Gakkai Zasshi 75, 1960-1971(1978). 